\title{
Three-Dimensional CFD Analysis of the Hand and Forearm in Swimming
}

\section{Daniel A. Marinho, António J. Silva, Victor M. Reis, Tiago M. Barbosa, João P. Vilas-Boas, Francisco B. Alves, Leandro Machado, and Abel I. Rouboa}

\begin{abstract}
The purpose of this study was to analyze the hydrodynamic characteristics of a realistic model of an elite swimmer hand/forearm using three-dimensional computational fluid dynamics techniques. A three-dimensional domain was designed to simulate the fluid flow around a swimmer hand and forearm model in different orientations $\left(0^{\circ}, 45^{\circ}\right.$, and $90^{\circ}$ for the three axes Ox, Oy and $\left.\mathrm{Oz}\right)$. The hand/forearm model was obtained through computerized tomography scans. Steady-state analyses were performed using the commercial code Fluent. The drag coefficient presented higher values than the lift coefficient for all model orientations. The drag coefficient of the hand/forearm model increased with the angle of attack, with the maximum value of the force coefficient corresponding to an angle of attack of $90^{\circ}$. The drag coefficient obtained the highest value at an orientation of the hand plane in which the model was directly perpendicular to the direction of the flow. An important contribution of the lift coefficient was observed at an angle of attack of $45^{\circ}$, which could have an important role in the overall propulsive force production of the hand and forearm in swimming phases, when the angle of attack is near $45^{\circ}$.
\end{abstract}

Keywords: computational fluid dynamics, aquatics, forces, drag, lift

The performance of swimmers is limited by their ability to produce effective propulsive force (the component of the total propulsive force acting in the direction of moving) and to minimize the drag forces resisting forward motion (Gardano \& Dabnichki, 2006; Marinho et al., 2009a). The measurement of the propulsive forces generated by a swimmer has been of interest to sports

Daniel A. Marinho is with the University of Beira Interior. Sport Sciences Department, Covilhã, Portugal, and the Centre of Research in Sports, Health and Human Development, Vila Real, Portugal). António J. Silva is with the Centre of Research in Sports, Health and Human Development, Vila Real, Portugal, and the University of Trás-os-Montes and Alto Douro, Vila Real, Portugal. Victor M. Reis is with the Centre of Research in Sports, Health and Human Development, Vila Real, Portugal, and the University of Trás-os-Montes and Alto Douro, Vila Real, Portugal. Tiago M. Barbosa is with the Centre of Research in Sports, Health and Human Development, Vila Real, Portugal and the Polytechnic Institute of Bragança, Bragança, Portugal. João P. Vilas-Boas is with the Faculty of Sport/CIFI2D, University of Porto, Porto, Portugal. Francisco B. Alves is with the Faculty of Human Kinetics. Technical University of Lisbon, Lisbon, Portugal. Leandro Machado is with the Faculty of Sport/CIFI2D, University of Porto, Porto, Portugal. Abel I. Rouboa (Corresponding Author) is with the Centre for Research and Technology of Agro-Environmental and Biology Sciences at UTAD, Vila Real, Portugal, and the University of Pennsylvania, Department of Mechanical Engineering and Applied Mechanics, Philadelphia, USA. biomechanics for many years. Despite the fact that the task of directly measuring the propulsive forces acting on a freely swimming subject is practically impossible, Hollander et al. (1986) developed a system for measuring active drag (MAD system) by determining the propulsive force applied to underwater push-off pads by a swimmer performing the front crawl arm action only. However, the intrusive nature of the device disables its use during competition and reduces its ecological validity (Payton \& Bartlett, 1995). A nonintrusive method of estimating propulsive hand forces during free swimming was developed by Schleihauf (1979) and was the basis of several studies (Berger et al., 1995; Sanders, 1999). In this method the instantaneous propulsive forces are estimated according to vectorial analysis of force combinations acting on model hands in an open-water channel and the recordings of underwater pulling action of a swimmer. Using a plastic resin model of an adult human hand, Schleihauf (1979) measured forces for known orientations to a constant water flow, determining drag and lift coefficients for specific orientations. These data were then used together with digitized kinematic data of the hand to estimate the lift, drag, and resultant force vectors produced during the stroke cycle of the swimmers. Regarding the water channel analysis, Schleihauf (1979) reported that lift coefficient values increased up to an attack angle around $40^{\circ}$ and then decreased, although some differences with respect to the sweepback angle were observed. Drag coefficient values increased with increasing the attack angle and were less sensitive to sweepback angle changes. 
Although these experiments accounted for fixture drag, the effects of interference drag at the wrist were not considered. Schleihauf (1979) assumed that the support rod encountered equal drag force whereas the hand model was attached or not. These researchers revealed the difficulties involved in conducting such studies experimentally. They had to choose between unwanted wave and ventilation drag or inaccurate interference drag (Bixler \& Riewald, 2002). In addition, Schleihauf (1979) neglected the axial component of the force, not considering the contribution of the forearm to swimming propulsion.

An alternative approach to evaluate the arm and hand swimming propulsion is to apply the numerical technique of computational fluid dynamics (CFD) instead of experimental methods to calculate the solution. Moreover, to avoid wave, ventilation, and interference drag, CFD has the advantage of showing detailed characteristics of fluid flow around the hand and arm.

The first application of CFD in swimming was conducted by Bixler and Schloder (1996), when they used a CFD two-dimensional analysis to evaluate the effects of accelerating a flat circular plate through water. The plate acceleration increased drag by $24 \%$ above nonaccelerating condition. Later on, Bixler and Riewald (2002) calculated drag and lift coefficient values using a three-dimensional hand/forearm model. Although only a $0^{\circ}$ of sweepback angle was analyzed, Bixler and Riewald (2002) showed the possibility of applying the three-dimensional CFD approach to swimming research. Additional research using CFD techniques was performed by Rouboa et al. (2006) to evaluate the steady and unsteady flow conditions of a swimmer's hand and arm. Authors reported similar results of those of Bixler and Schloder (1996), even if a two-dimensional model were used. Nevertheless, only recently (Bixler et al., 2007), the validity and accuracy of the CFD analysis as a tool to examine the water flow and the hydrodynamic forces around the human body was confirmed, in the analysis of the hydrodynamic drag force during the underwater gliding of a whole swimmer model.

In the current study we attempted to improve previous investigations applying CFD to analyze the hydrodynamic characteristics of the human hand/forearm. In one sense, CFD has shown to be a complementary tool to experimental tests, allowing to correct some concerns of those experiments (e.g., interference, ventilation, and/ or wave drag). In another sense, we aimed to test drag and lift coefficients of the hand and forearm in different orientation angles, testing not only a $0^{\circ}$ sweepback angle, as Bixler and Riewald (2002) did, but also testing $90^{\circ}$, $180^{\circ}$, and $270^{\circ}$ sweepback orientations.

Furthermore, the reserve engineering procedures were applied to obtain a realistic model of an elite male swimmer's hand and forearm (Marinho et al., 2010). It was hypothesized that these data could supply important results to be applied to real motions of elite level swimmers, considering different arm orientations usually adopted during swimming. To our knowledge, there is no research published using a numerical approach on the repercussion of different sweepback angles (rather than $0^{\circ}$ ) and with anthropometrical data of elite swimmers' hand/forearm.

Therefore, the purpose of the current study was to analyze the hydrodynamic characteristics of a realistic model of an elite swimmer hand/forearm using steadystate computational fluid dynamics.

\section{Methods}

\section{Digital Model of the Swimmer Hand and Forearm}

A CFD model was created based upon an Olympic swimmer's right forearm and hand. The hand/arm boundary was located at the level of the styloid processes of the radius and ulna. The model was created by computer tomography scans of a male swimmer's hand and forearm, allowing the acquisition of the boundaries of the human segments (Marinho et al., 2010). The subject was an Olympic-level swimmer, who participated in the 2004 Olympic Games, in Athens. This protocol has been approved by the appropriate ethical committee of the institution in which it was performed, and the subject gave informed consent to participate in this work.

Cross-sectional scans of the right hand and forearm were conducted using a Toshiba Aquilion 4 computer tomography scanner (Marinho et al., 2010). The subject was lying prone, with his right arm extended ahead and fully pronated. This procedure was conducted with the thumb adducted into the plane of the hand and with the fingers closed together. The image processing programs used in this study were the Anatomics Pro (Anatomics, Kannapolis, NC, USA) and the software FreeForm (SensAble Technologies, Woburn, MA, USA).

The data were converted into an IGES format (*.igs) that could be read by the grid generator Gambit/Fluent (Fluent Inc, Hanover, NH, USA) to define the finite elements approach through the three-dimensional surfaces. This geometry protruded into a dome-shaped mesh of fluid cells from its base, which was in the plane of the dome base.

\section{Mathematical Model}

The dynamic fluid forces produced by the hand and forearm segment, lift (L) and drag (D), were calculated in this study. Drag force is defined as the force acting parallel to the flow direction and lift force lies perpendicular to the drag force. These forces were computed by the application of the Equations 1 and 2 (Sanders, 1999; Schleihauf, 1979).

$$
\begin{aligned}
& D=1 / 2 C_{D} \rho S v^{2} \\
& L=1 / 2 C_{L} \rho S v^{2}
\end{aligned}
$$


In Equations 1 and 2, $C_{D}$ and $C_{L}$ represent the drag and lift coefficients, respectively, $v$ represents the water velocity, $\rho$ represents the fluid density and $S$ represents the projection surface of the model for different angles of attack used in this study.

The numerical simulation techniques methodology consists of a mathematical model applied to the fluid flow in a given domain that replaces the Navier-Stokes equations with discretized algebraic expressions. These equations can be solved by iterative computerized calculations. The Fluent CFD code (Fluent, Inc.) was used to develop and solve these equations using the finite volume approach, where the equations were integrated over each control volume. The domain consists of a threedimensional grid or mesh of cells that simulate the fluid flow around the human segments.

We used the segregated solver with the standard k-epsilon turbulence model because this model was shown to be accurate in previous research (Bixler \& Riewald, 2002; Bixler et al., 2007; Moreira et al., 2006). A criterion of convergence of $10^{-5}$ for the residuals of the various flow parameters was used (Zaidi et al., 2008). The considered fluid was water, with a turbulence intensity of $1.0 \%$ and a turbulence scale of $0.10 \mathrm{~m}$. The water density was $998.2 \mathrm{~kg} \cdot \mathrm{m}^{-3}$ with a viscosity of $0.001 \mathrm{~kg} \cdot(\mathrm{m} \cdot \mathrm{s})^{-1}$ (Bixler \& Riewald, 2002).

\section{Boundary Conditions}

The numerical simulations were carried out in three dimensions for the computational domain in steady flow. A three-dimensional domain was designed to simulate the fluid flow around a swimmer's hand and forearm model
(Figure 1). The whole domain was meshed with 900,000 cells. The grid was a hybrid mesh composed of prisms and pyramids. By decreasing the grid node separation in areas of high velocity and pressure gradients, the model was made more accurate (Bixler et al., 2007; Marinho et al., 2010).

The angle of attack is defined as the angle between the hand/forearm and the flow direction, and the sweepback angle is defined as the leading edge of the hand relative to the fluid flow. Angles of attack of hand/ forearm models of $0^{\circ}, 45^{\circ}$, and $90^{\circ}$, with sweepback angles of $0^{\circ}$ (thumb as the leading edge), $90^{\circ}$ (top of the fingers as the leading edge), and $180^{\circ}$ (little finger as the leading edge) were used for the calculations (Schleihauf, 1979).

Water velocity was prescribed to the inlet portion of the dome surface and was held steady at values between $0.50 \mathrm{~m} \cdot \mathrm{s}^{-1}$ and $4.00 \mathrm{~m} \cdot \mathrm{s}^{-1}$, with $0.50 \mathrm{~m} \cdot \mathrm{s}^{-1}$ increments as reported by experimental literature for swimmer's upper limb actions (Lauder et al., 2001).

The dome's base was a plane of symmetry, requiring the flow there to remain in that plane. Around the model, the velocity was considered as equal to zero. This allows the adhesion of the fluid to the model.

The independent variables were the angle of attack, sweepback angle, and inlet flow velocity. The dependent variables were pressure and velocity of the fluid within the dome. Postprocessing of the results with Fluent allowed the calculation of component forces through integration of pressures on the hand/forearm surfaces. Based on the computed forces on the hand/forearm models drag $\left(C_{D}\right)$ and lift $\left(\mathrm{C}_{\mathrm{L}}\right)$ coefficients were determined, using Equations 1 and 2.

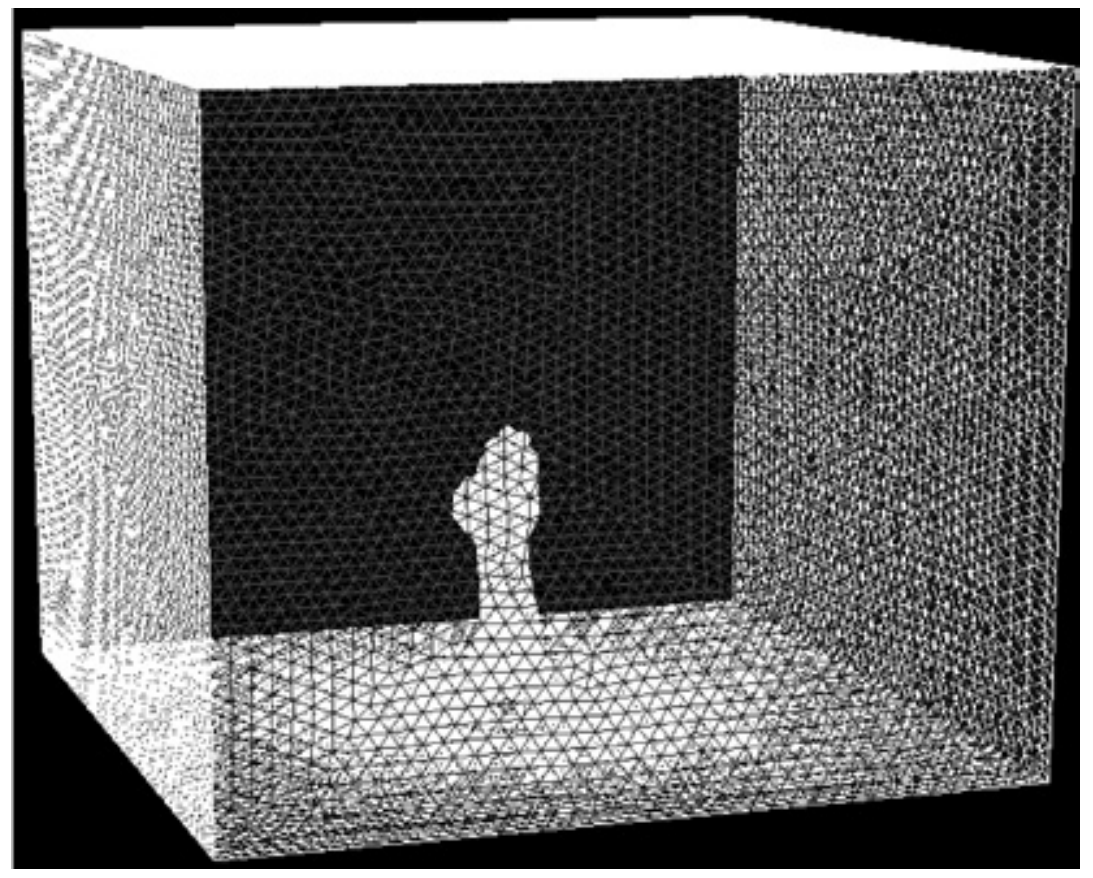

Figure 1 - Hand and forearm model inside the three-dimensional domain. The whole domain was meshed with 900,000 cells. 


\section{Results}

The path of the water moving near the hand and forearm surface can be revealed by a CFD oil-film plot. In Figure 2 the flow path line at a $90^{\circ}$ angle of attack of the hand and forearm segment is presented. One can observe the direction of the water flow around the propelling segments.

In Figure 3 the evolution of the values of the combined hand and forearm $C_{D}$ and $C_{L}$ according to flow velocity for a sweepback angle of $0^{\circ}$ is presented. For a sweepback angle of $0^{\circ}$ (a similar tendency was observed for sweepback angles of $90^{\circ}$ and $180^{\circ}$, although not presented in this figure), the $C_{D}$ and $C_{L}$ remained almost constant regarding the different flow velocities. Nevertheless, we were able to note a slight decrease in the force coefficients, especially from 0.50 to $2.0 \mathrm{~m} \cdot \mathrm{s}^{-1}$. This situation occurred for a given angle of attack and with the same tendency at sweepback angles of $0^{\circ}, 90^{\circ}$, and $180^{\circ}$.

Moreover, the $\mathrm{C}_{\mathrm{D}}$ was the coefficient that accounts more for the hand and forearm propulsion, presenting higher values than the $\mathrm{C}_{\mathrm{L}}$ for the entire model orientations. The $\mathrm{C}_{\mathrm{D}}$ of the hand/forearm model increased with the angle of attack (Figure 4 ). The $C_{D}$ presented the maximum values with an angle of attack of $90^{\circ}$ for the three sweepback angles $\left(C_{D} \approx 0.90\right)$ and the minimum values with an angle of attack of $0^{\circ}\left(\mathrm{C}_{\mathrm{D}} \approx 0.45\right.$, sweepback angle $=0^{\circ}, 180^{\circ} ; \mathrm{C}_{\mathrm{D}} \approx 0.20$, sweepback angle $=90^{\circ}$ ). The $\mathrm{C}_{\mathrm{L}}$ of the model presented the maximum values with an angle of attack of $45^{\circ}\left(\mathrm{C}_{\mathrm{L}} \approx 0.50\right.$, sweepback angle $=180^{\circ} ; \mathrm{C}_{\mathrm{L}} \approx 0.30$, sweepback angle $=0^{\circ}, 90^{\circ}$; Figure 5 ). The values of $C_{L}$ were very similar for the angles of attack of $0^{\circ}$ and $90^{\circ}$.

As can be observed in Figures 4 and 5 , the $C_{D}$ and $C_{L}$ of the hand/forearm model followed the same tendency in relation with the angle of attack in the three sweepback angles that were tested. However, it seems interesting to reinforce the great value of the $\mathrm{C}_{\mathrm{L}}$ at an angle of attack of $45^{\circ}$, which could have an important contribution to the overall propulsive force production of the hand and forearm in swimming technique, especially when the

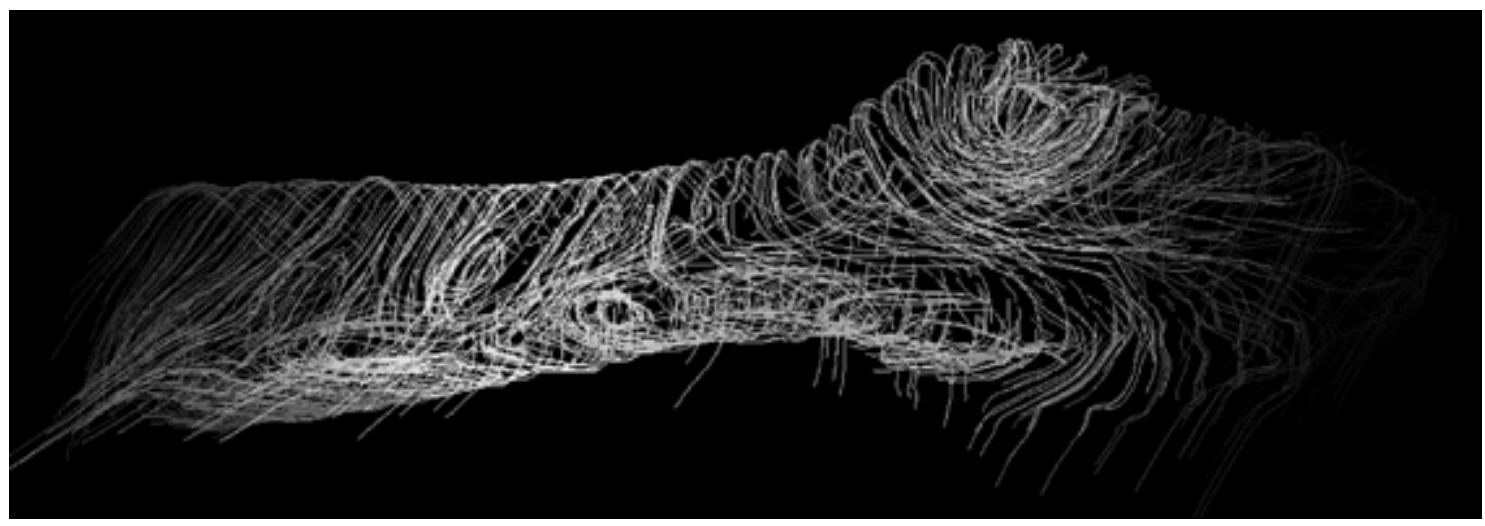

Figure 2 - Computational fluid dynamics oil-film plot shows the direction of the water flow around the model. The flow path line at a $90^{\circ}$ angle of attack of the hand and forearm segment is presented (sweepback angle $=0^{\circ}$; flow velocity $=2.0 \mathrm{~m} \cdot \mathrm{s}^{-1}$ ).

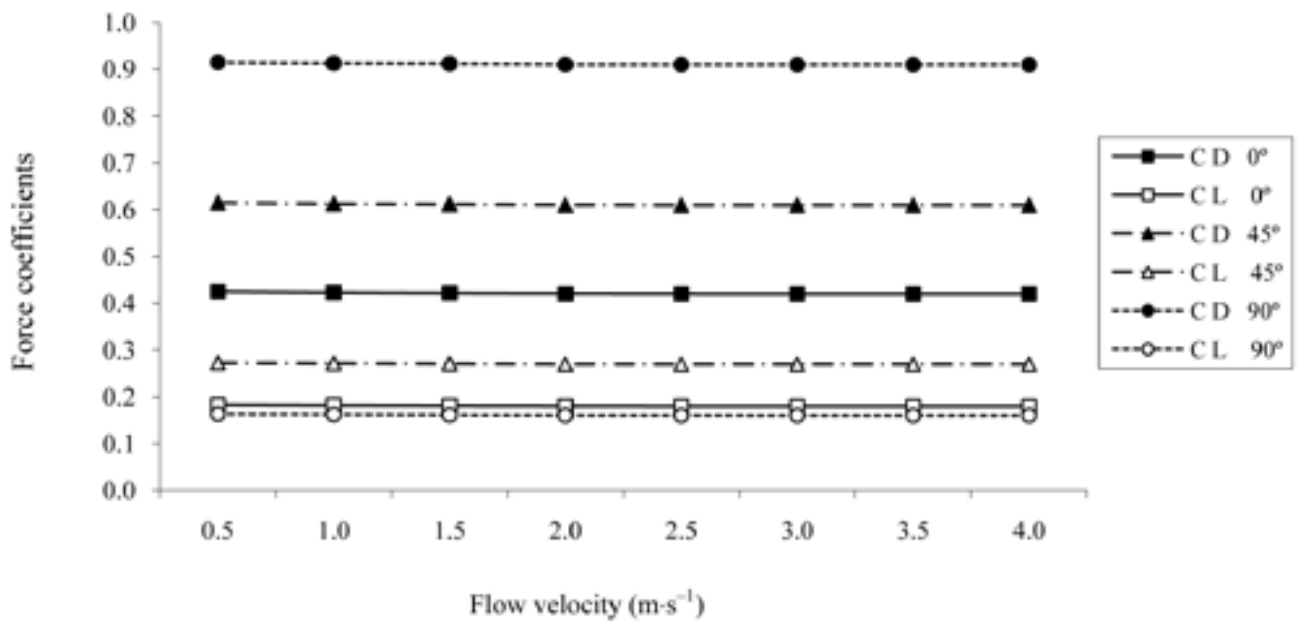

Figure 3 - Drag and lift coefficients vs. flow velocity for each angle of attack $\left(0^{\circ}, 45^{\circ}\right.$, and $\left.90^{\circ}\right)$. Sweepback angle $=0^{\circ}$. 


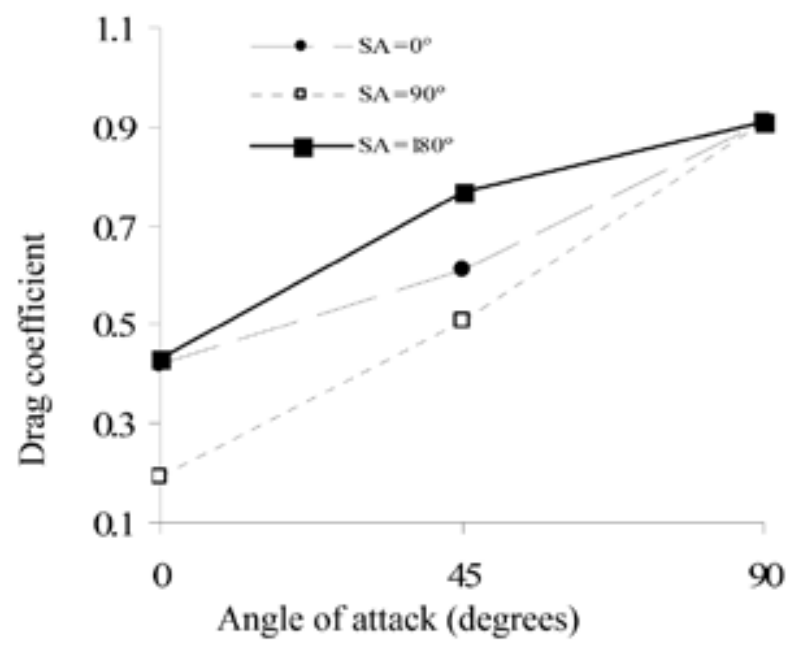

Figure 4 - Drag coefficient vs. angle of attack for each sweepback angle (SA). Flow velocity $=2.0 \mathrm{~m} \cdot \mathrm{s}^{-1}$.

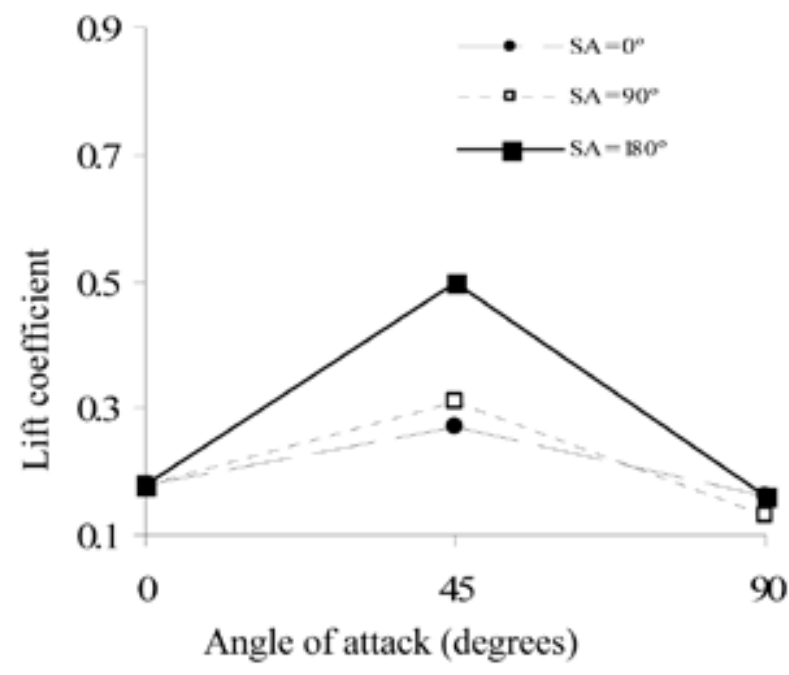

Figure 5 - Lift coefficient vs. angle of attack for each sweepback angle $(\mathrm{SA})$. Flow velocity $=2.0 \mathrm{~m} \cdot \mathrm{s}^{-1}$.

little finger leads the motion (sweepback angle $=180^{\circ}$ ). Regarding $\mathrm{C}_{\mathrm{D}}$, the major differences occurred as well with an angle of attack of $45^{\circ}$, when the sweepback angle is $180^{\circ}$.

\section{Discussion}

The aim of this study was to analyze the hydrodynamic characteristics of a realistic model of an elite swimmer hand/forearm using CFD. The main result was that the drag coefficient presented higher values than the lift coefficient for the entire model orientation, although an important contribution of the lift coefficient was observed at an angle of attack of $45^{\circ}$.
Computational fluid dynamics methodology seems to be a reliable and valid method to be used in swimming hydrodynamic research (Bixler et al., 2007). Bixler et al. (2007) compared total drag forces among a real swimmer, a digital CFD model of this same swimmer, and a mannequin based on the digital model. The authors found drag forces determined from the digital model using the CFD approach to be within $4 \%$ of the values assessed experimentally for the mannequin, although the mannequin drag was found to be $18 \%$ less than the real swimmer drag.

In the current study, we tried to improve the previous CFD analysis, using a real model of the swimmer hand and forearm. Furthermore, it is used different orientation angles of the hand and forearm segment. Changes in the sweepback angle were included in the simulations, trying to approach the different upper arm orientations often adopted during actual swimming. In addition, the use of a plane of symmetry in the dome's base was conducted to ensure that the flow remained in that plane. Although this is an approximation to actually modeling an elbow and upper arm, it avoids the edge effects that would have occurred if water were allowed to flow under the bottom of the arm, or the wave and ventilation drag that would have occurred if the dome bottom were modeled as a free water surface (Bixler \& Riewald, 2002).

For the three sweepback angles, the $C_{D}$ and $C_{L}$ values remained almost constant throughout the flow velocities tested. A similar situation was already reported in other numerical studies (Bixler \& Riewald, 2002; Silva et al., 2005; Rouboa et al., 2006; Alves et al., 2007; Marinho et al., 2009b). Nevertheless, we were able to note a slightly decrease in the force coefficients, especially from 0.50 to $2.0 \mathrm{~m} \cdot \mathrm{s}^{-1}$. Berger et al. (1995) in a towing tank and Bixler and Riewald (2002) in a numerical study reported both a similar situation for lower velocities. A little decrease in the force coefficient values occurred with the velocity increase. However, from a practical standpoint, the coefficients were considered independent of the flow velocity. This finding seems to be consistent with the theory of fluid mechanics. As the Reynolds number increases (due to velocity increase), $C_{D}$ values decrease up to a critical value of Reynolds number above which $C_{D}$ values remain almost constant (Taiar et al., 1999; Vogel, 1994). In swimming conditions (Taiar et al., 1999) and also in fluid mechanics experiments (Massey, 1989), it is expected that the decrease in $C_{D}$ values at a higher Reynolds number should be reduced.

The $C_{D}$ presented higher values than the $C_{L}$ for the entire model orientations. The $C_{D}$ of the hand/forearm model increased with the angle of attack, with the maximum value of force production corresponding to an angle of attack of $90^{\circ}$. The $C_{D}$ obtained the highest value at an orientation of the hand plane where the model was directly perpendicular to the direction of the flow. The same result was reported by other authors (Berger et al., 1995; Marinho et al., 2010; Schleihauf, 1979), in which the drag force increased to a maximum where the plane was the same as the presented in this work (angle of attack $\left.=90^{\circ}\right)$. 
In this position, the hand area presented the maximum value, creating an effective resisting area (Schleihauf, 1979), allowing increases of the $C_{D}$ (equation 1 ).

The $\mathrm{C}_{\mathrm{L}}$ of the hand and forearm segment presented lower values for angles of attack of $0^{\circ}$ and $90^{\circ}$, but presented higher values at an angle of attack of $45^{\circ}$, especially when the little finger leads the motion (sweepback angle $=180^{\circ}$ ), which could lead us to speculate that lift force can play an important role in similar hand/forearm orientations during swimming. This fact may be related to the differences in the flow around the hand when the leading edge is the little finger. In this position it is possible that a low-pressure area on the knuckle side of the hand is created, producing more lift and a smoother flow around the hand.

Bixler and Riewald (2002) reported that forearm drag was essentially constant $\left(C_{D} \approx 0.65\right)$ and forearm lift was almost null. Furthermore, hand drag presented the minimum value near angle of attack of $0^{\circ}$ and the maximum value was obtained near $90^{\circ}\left(C_{D} \approx 1.15\right)$. Hand lift was almost null at $90^{\circ}$ and peaked near $60^{\circ}\left(C_{L} \approx 0.60\right)$. Combined hand and forearm drag presented the highest value at $90^{\circ}\left(\mathrm{C}_{\mathrm{D}} \approx 0.90\right)$ and the minimum value at $0^{\circ}$ $\left(C_{D} \approx 0.30\right)$, whereas combined hand and forearm lift presented the highest values in the range of $30-60^{\circ}\left(C_{L}\right.$ $\approx 0.30)$ and the minimum value at $90^{\circ}\left(\mathrm{C}_{\mathrm{L}} \approx 0\right)$. At the same sweepback angle, our results are similar to the ones of Bixler and Riewald (2002). Considering the Schleihauf (1979) data, it is difficult to compare our results, since this author only analyzed a hand model. Nevertheless, considering the results of Bixler and Riewald (2002) one can notice that the experimental values of Schleihauf (1979) are higher than the CFD data. This fact might be due to flume interference, which increases lift and especially drag. Depending on the hand orientation, Kudo et al. (2008) reported that this interference effect could increase the $C_{D}$ and the $C_{L}$ up to $98 \%$ and $12 \%$, respectively. This finding could once again suggest the important contribution that CFD can play in future hydrodynamic research.

Further research during actual swimming is necessary to establish the orientation and movement of the hand in which the forward component of the sum of drag and lift forces is maximal. Moreover, since distinct patterns of timing and sequence of body roll on front crawl and backstroke are used by the swimmers (Payton et al., 2002), the effect of this rotation on the propulsive force production should be simulated. Considering these results, it seems essential to analyze a larger range of angles of attack, trying to clarify the true importance of the lift force to the propulsive force production. The $\mathrm{C}_{\mathrm{D}}$ has its maximum values if the flow vector is at right angles to the hand plane, whereas the $\mathrm{C}_{\mathrm{L}}$ has its maximum values if the hand plane makes an angle with the flow vector. It seems probable that the lift force plays an important role at angles of attack other than the $45^{\circ}$, as is suggested by Schleihauf (1979) at an attack angle of $15^{\circ}$. Moreover, one interesting issue that should be carefully analyzed is related to the turbulent model used during CFD simulations. Most studies in swimming, including this one, used the k-epsilon turbulent model. A recent study reported that the standard k-omega model accurately predicts the drag forces of the swimmer body while the standard k-epsilon model underestimates these values (Zaidi et al., 2010). However, in the current study only a hand/forearm segment was computed thus, some extra research should be performed in the future.

In summary, the $C_{D}$ presented always the higher values for the hand/forearm segment with a maximum value at an angle of attack of $90^{\circ}$. The $C_{L}$ seems to play an important role at an angle of attack of $45^{\circ}$, especially when the little finger leads the motion. These data confirm recent studies reporting supremacy of drag component and an important contribution of lift force to the overall propulsive force generation by the hand/forearm in swimming phases, when the angle of attack nears $45^{\circ}$ (e.g., Alves et al., 2007; Marinho et al., 2009b).

The results have demonstrated that this numerical tool can effectively be used both to improve the foundational knowledge of swimming hydrodynamics. Ideally, with increases in the database of information created by the CFD analysis, more conclusions can be derived that could be applicable to larger swimming populations. Further studies should include the unsteady effects of motion, such as accelerations and multiaxis rotations. This could be accomplished by performing transient time-dependent analysis using user-defined functions and moving meshes (Lyttle \& Keys, 2006). The ultimate goal should be to use this numerical technique to evaluate complete arm and leg strokes and to prescribe the optimum pulling pattern.

\section{Acknowledgments}

This work was supported by the Portuguese government by a grant of the Science and Technology Foundation (SFRH/ BD/25241/2005; PTDC/DES/098532/2008). We would like to acknowledge the Radiology Department of Hospital de São João, in Porto, Portugal, and personally to the department director, Isabel Ramos. We would also like to express our gratitude to the Med Mat Innovation Company, in Maia, Portugal—especially to José Domingos Santos and the Bruno Sá.

\section{References}

Alves, F., Marinho, D., Leal, L., Rouboa, A., \& Silva, A. (2007). 3-D computational fluid dynamics of the hand and forearm in swimming. Medicine and Science in Sports and Exercise, 39(Suppl. 1), S9.

Berger, M.A.M., de Groot, G., \& Hollander, P. (1995). Hydrodynamic drag and lift forces on human hand/arm models. Journal of Biomechanics, 28(2), 125-133.

Bixler, B., \& Riewald, S. (2002). Analysis of swimmer's hand and arm in steady flow conditions using computational fluid dynamics. Journal of Biomechanics, 35(5), 713-717.

Bixler, B., \& Schloder, M. (1996). Computational fluid dynamics: an analytical tool for the 21st century swimming scientist. Journal of Swimming Research, 11(1), 4-22.

Bixler, B., Pease, D., \& Fairhurst, F. (2007). The accuracy of computational fluid dynamics analysis of the passive drag of a male swimmer. Sports Biomechanics, 6(1), 81-98. 
Gardano, P., \& Dabnichki, P. (2006). On hydrodynamics of drag and lift of the human arm. Journal of Biomechanics, 39(15), 2767-2773.

Hollander, A.P., de Groot, G., Van Ingen Schenau, G.L., Toussaint, H.M., de Best, H., Peeters, W., et al. (1986). Measurement of active drag during crawl arm stroke swimming. Journal of Sports Sciences, 4(1), 21-30.

Kudo, S., Vennel, R., Wilson, B., Waddell, N., \& Sato, Y. (2008). Influence of surface penetration on measured fluid force on a hand model. Journal of Biomechanics, 41, 3502-3505.

Lauder, M., Dabnichki, P., \& Bartlett, R. (2001). Improved accuracy and reliability of sweepback angle, pitch angle and hand velocity calculations in swimming. Journal of Biomechanics, 34(1), 31-39.

Lyttle, A., \& Keys, M. (2006). The application of computational fluid dynamics for technique prescription in underwater kicking. Portuguese Journal of Sport Sciences, 6(Suppl. 2), 233-235.

Marinho, D.A., Reis, V.M., Alves, F.B., Vilas-Boas, J.P., Machado, L., Silva, A.J., et al. (2009a). The hydrodynamic drag during gliding in swimming. Journal of Applied Biomechanics, 25(3), 253-257.

Marinho, D.A., Barbosa, T.M., Reis, V.M., Kjendlie, P.L., Alves, F.B., Vilas-Boas, J.P., et al. (2010). Swimming propulsion forces are enhanced by a small finger spread. Journal of Applied Biomechanics, 26(1), 87-92.

Marinho, D.A., Rouboa, A.I., Alves, F.B., Vilas-Boas, J.P., Machado, L., Reis, V.M., et al. (2009b). Hydrodynamic analysis of different thumb positions in swimming. Journal of Sports Science and Medicine, 8(1), 58-66.

Massey, B.S. (1989). Mechanics of Fluids. London: Chapman \& Hall.

Moreira, A., Rouboa, A., Silva, A.J., Sousa, L., Marinho, D., Alves, F., et al. (2006). Computational analysis of the turbulent flow around a cylinder. Portuguese Journal of Sport Sciences, 6(Suppl. 1), 105.
Payton, C., \& Bartlett, R. (1995). Estimating propulsive forces in swimming from three-dimensional kinematic data. Journal of Sports Sciences, 13(6), 447-454.

Payton, C., Baltzopoulos, V., \& Bartlett, R. (2002). Contributions of rotations of the trunk and upper extremity to hand velocity during front crawl swimming. Journal of Applied Biomechanics, 18(3), 243.

Rouboa, A., Silva, A., Leal, L., Rocha, J., \& Alves, F. (2006). The effect of swimmer's hand/forearm acceleration on propulsive forces generation using computational fluid dynamics. Journal of Biomechanics, 39(7), 1239-1248.

Sanders, R.H. (1999). Hydrodynamic characteristics of a swimmer's hand. Journal of Applied Biomechanics, 15(1), 3-26.

Schleihauf, R.E. (1979). A hydrodynamic analysis of swimming propulsion. In J. Terauds \& E.W. Bedingfield (Eds.), Swimming III (pp. 70-109). Baltimore: University Park Press.

Silva, A., Rouboa, A., Leal, L., Rocha, J., Alves, F., Moreira, A., et al. (2005). Measurement of swimmer's hand/forearm propulsive forces generation using computational fluid dynamics. Portuguese Journal of Sport Sciences, 5(3), 288-297.

Taiar, R., Sagnes, P., Henry, C., Dufour, A.B., \& Rouard, A.H. (1999). Hydrodynamics optimization in butterfly swimming: position, drag coefficient and performance. Journal of Biomechanics, 32, 803-810.

Vogel, S. (1994). Life in Moving Fluids. Princeton: Princeton University Press.

Zaidi, H., Fohanno, S., Taiar, R., \& Polidori, G. (2010). Turbulence model choice for the calculation of drag forces when using the CFD method. Journal of Biomechanics, 43, 405-411.

Zaidi, H., Taiar, R., Fohanno, S., \& Polidori, G. (2008). Analysis of the effect of swimmer's head position on swimming performance using computational fluid dynamics. Journal of Biomechanics, 41, 1350-1358. 